\title{
Resonant Drag Instability of Grains Streaming in Fluids
}

\author{
J. Squire ${ }^{1,2}$ (1) and P. F. Hopkins ${ }^{1,2}$ (i) \\ ${ }^{1}$ Theoretical Astrophysics, 350-17, California Institute of Technology, Pasadena, CA 91125, USA; jsquire@caltech.edu \\ ${ }^{2}$ Walter Burke Institute for Theoretical Physics, Pasadena, CA 91125, USA \\ Received 2018 February 20; accepted 2018 March 8; published 2018 March 23
}

\begin{abstract}
We show that grains streaming through a fluid are generically unstable if their velocity, projected along some direction, matches the phase velocity of a fluid wave (linear oscillation). This can occur whenever grains stream faster than any fluid wave. The wave itself can be quite general-sound waves, magnetosonic waves, epicyclic oscillations, and Brunt-Väisälä oscillations each generate instabilities, for example. We derive a simple expression for the growth rates of these "resonant drag instabilities" (RDI). This expression (i) illustrates why such instabilities are so virulent and generic and (ii) allows for simple analytic computation of RDI growth rates and properties for different fluids. As examples, we introduce several new instabilities, which could see application across a variety of physical systems from atmospheres to protoplanetary disks, the interstellar medium, and galactic outflows. The matrix-based resonance formalism we introduce can also be applied more generally in other (nonfluid) contexts, providing a simple means for calculating and understanding the stability properties of interacting systems.
\end{abstract}

Key words: instabilities - ISM: kinematics and dynamics - magnetohydrodynamics (MHD) - planets and satellites: formation - stars: general - turbulence

\section{Introduction}

Many astrophysical fluids-e.g., the interstellar medium (Ferrière 2001), disks (Armitage 2011), and our solar system (Krüger et al. 2015) — are laden with solid grains, or dust. Because they contain a large proportion of the available metals, dust is fundamental to key astrophysical processes, such as star and planet formation (see, e.g., Käufl \& Siebenmorgen 1996; Draine 2004; Chiang \& Youdin 2010). It is thus crucial to understand dust-fluid dynamics (Saffman 1962; Pumir \& Wilkinson 2016): how the phases interact through drag and/or Lorentz forces; what forces influence the fluid but not dust grains, and vice versa.

In this Letter, we ask a simple question: if dust grains stream through a fluid with some constant relative velocity $\mathbf{w}_{s}$, is the coupled system stable? We show, quite generally, that this system is usually unstable if the phase speed of a wave in the fluid matches the projection of $\boldsymbol{w}_{s}$ along the wave propagation direction. This resonant fluid wave is stationary in the frame of the dust grains and couples very efficiently to grain density perturbations. This usually renders the wave unstable because it can feed off the energy in the background drift, causing clumping of the grains in space as the instability grows. Many fluid waves-e.g., sound waves, magnetosonic waves, BruntVäisälä oscillations, or epicyclic oscillations — can cause such a "resonant drag instability" (RDI). Furthermore, because the fluid wave can be destabilized at an angle to the grain's velocity $\mathbf{w}_{s}$, any streaming motion faster than the phase speed can cause an RDI. For example, in hydrodynamics, the RDI occurs whenever $w_{s}=\left|\mathbf{w}_{s}\right|>c_{s}$ (the sound speed), while in magnetohydrodynamics (MHD) or a stratified fluid, the RDI is possible for any $w_{s}$.

A relative dust-to-gas streaming velocity $\mathbf{w}_{s}$ can occur for a variety of reasons. In many astrophysical systems-e.g., near active galactic nucleii (Krolik \& Begelman 1988; Thompson et al. 2005; Hopkins et al. 2012), in the envelopes of cool stars (Dominik et al. 1989; Norris et al. 2012), or near star-forming regions (Franco et al. 1991; Murray et al. 2005)—radiation pressure more strongly affects the dust grains than the gas. As grains are accelerated, they drag the gas with them, reaching a terminal $\mathbf{w}_{s}$ when the drag force nearly balances the radiative force (Gilman 1972; Netzer \& Elitzur 1993). Another source of relative drift occurs when the fluid-but not the dust-is supported by thermal pressure against gravity. In, for example, planetary atmospheres or astrophysical disks, this causes grains to settle in the direction of gravity (Goldreich \& Ward 1973; Nakagawa et al. 1986). However, despite these diverse mechanisms that cause a relative drift, in each case, the stability of the coupled dust-gas system can be calculated in the frame where the fluid is stationary (a bulk velocity or linear acceleration does not change the system's spectral stability properties; Hopkins \& Squire 2017). Thus, in this Letter we simply prescribe $\mathbf{w}_{s}$, remaining agnostic about its origin. We also assume a homogenous background gas and dust density (the local approximation), neglect dissipative processes (e.g., viscosity) in the fluid, and assume that grains interact with the gas only through drag forces (neglecting, e.g., grain charge and dusty plasma effects; Rao et al. 1990; Shukla 2001; Weingartner \& Draine 2001; Draine 2004). Detailed physical applications are treated in companion papers (Hopkins \& Squire 2017, 2018; Squire \& Hopkins 2017); the purpose of this Letter is to introduce the basic mathematical formalism and structure of dust-gas RDIs.

Following a general derivation of the RDI, this Letter is organized into three examples: hydrodynamics, MHD, and stratified fluids. The general nature of these instabilities has not (to our knowledge) been discussed in previous works, though specific manifestations of the hydrodynamic instability are studied in Morris (1993), Mastrodemos et al. (1996), and Deguchi (1997), and instabilities of a streaming neutral gas in MHD are treated in detail in Tytarenko et al. (2002). We also note that the widely studied "streaming instability" of grains in protoplanetary disks (Goodman \& Pindor 2000; Youdin \& Goodman 2005; Johansen et al. 2007) is an RDI with disk epicyclic oscillations, although its resonant nature has not (to our knowledge) been recognized previously. Similar ideas are more generally related to a variety of instabilities in fluids and 
plasmas (e.g., Kennel \& Wong 1967; Childress \& Spiegel 1975; Sundaresan 2003; Verscharen et al. 2013). Throughout this Letter, we study the RDI only exactly at resonance, though each example also displays an array of other slower-growing instabilities (see Hopkins \& Squire 2017; as shown below, resonant modes are always the fastest growing at low grain concentrations).

\section{Basic Theory of Resonance Instability}

Before deriving the RDI dispersion relation for various specific fluid systems, we consider the mathematics of interacting linear systems. Our purpose here is twofold: first, these results show why resonances generically lead to virulent instabilities; second, we will derive formulae for the RDI growth rate in terms of fluid eigenmodes (Equations (4) and (6)). These formulae allow the dispersion relation of different RDIs to be calculated with relative ease, even for complicated fluid systems (e.g., MHD in 3D). Aspects of these results are related to "Krein collisions" in the theory of Hamiltonian mechanics (Krein 1950; Kirillov 2013), although we do not restrict ourselves to Hamiltonian systems.

Consider an arbitrary system of equations that describes the motion of a coupled system of fluid, denoted $f$ (e.g., with density and velocity variables, $\boldsymbol{f}=(\rho, \boldsymbol{u}, \ldots))$, and dust, $\boldsymbol{a}=\left(\rho_{d}, \boldsymbol{v}\right)$ (the dust continuum density and velocity). For small perturbations $(\boldsymbol{f}=\langle\boldsymbol{f}\rangle+\delta \boldsymbol{f}, \boldsymbol{a}=\langle\boldsymbol{a}\rangle+\delta \boldsymbol{a})$, which are Fourier decomposed in space and time $\left(\delta g(\boldsymbol{x}, t)=\delta g e^{i \boldsymbol{k} \cdot \boldsymbol{x}-i \omega t}\right)$, the linearized equations of motion (see Equations (7)-(8) below) take the form of a generic eigenvalue problem,

$$
\begin{aligned}
\omega\left(\begin{array}{l}
\delta \boldsymbol{a} \\
\delta \boldsymbol{f}
\end{array}\right) & =\mathbb{T}\left(\begin{array}{l}
\delta \boldsymbol{a} \\
\delta \boldsymbol{f}
\end{array}\right)=\left(\mathbb{T}_{0}+\mu \mathbb{T}^{(1)}\right)\left(\begin{array}{l}
\delta \boldsymbol{a} \\
\delta \boldsymbol{f}
\end{array}\right), \\
\mathbb{T}_{0} & =\left(\begin{array}{ll}
\mathcal{A} & \mathcal{C} \\
0 & \mathcal{F}
\end{array}\right), \quad \mathbb{T}^{(1)}=\left(\begin{array}{cc}
\mathcal{T}_{A A}^{(1)} & \mathcal{T}_{A F}^{(1)} \\
\mathcal{T}_{F A}^{(1)} & \mathcal{T}_{F F}^{(1)}
\end{array}\right) .
\end{aligned}
$$

Here $\mathbb{T} \equiv \mathbb{T}_{0}+\mu \mathbb{T}^{(1)}$ is the full linearized system of equations, decomposed (without loss of generality) into the block-matrix form $\mathbb{T}_{0}$ (composed of $\mathcal{A}, \mathcal{F}, \mathcal{C}$ ) and $\mu \mathbb{T}^{(1)}$ (where $\mu \equiv \rho_{d} / \rho$ is the ratio of dust to fluid continuum densities). Submatrix $\mathcal{F}$ describes the fluid in the absence of dust, $\mathcal{A}$ describes dust in the absence of fluid motions, $\mathcal{C}$ couples the dust to the fluid (e.g., drag on the dust), and $\mu \mathbb{T}^{(1)}$ contains any coupling of the fluid to the dust (e.g., the back-reaction from dust, in $\mathcal{T}_{F A}^{(1)}$ ). If $\operatorname{Im}(\omega)>0$, the system is unstable (perturbations grow).

Now stipulate that $\mathcal{A}$ and $\mathcal{F}$ share an eigenvalue, $\omega=\omega_{0}$, which we define as a resonance. It is most instructive to examine the limit $\mu \ll 1$, i.e., to ask what happens to the eigenvalue $\omega_{0}$ as the dust starts influencing the fluid's dynamics. Mathematically, this is the eigenvalue perturbation, $\omega=\omega_{0}+\omega^{(1)}+\ldots$, due to $\mu \mathbb{T}^{(1)}$. Assuming $\omega_{0}$ is a semisimple eigenvalue of $\mathcal{A}$ and $\mathcal{F}$ individually, define its right and left eigenvectors,

$$
\left(\mathcal{A}-\omega_{0} \mathbb{I}\right) \xi_{\mathcal{A}}^{R}=0 \quad \text { and } \quad \xi_{\mathcal{A}}^{L}\left(\mathcal{A}-\omega_{0} \mathbb{I}\right)=0,
$$

with $\xi_{\mathcal{A}}^{L} \xi_{\mathcal{A}}^{R}=1, \mathbb{I}$ the identity matrix, and equivalent definitions for $\mathcal{F}$ with $\xi_{\mathcal{F}}^{L, R}$. Using the block structure of $\mathbb{T}_{0}$ (Dobson et al. 2001), one can show that if $\xi_{\mathcal{A}}^{L} \mathcal{C} \xi_{\mathcal{F}}^{R} \neq 0$, then $\omega_{0}$ is a defective eigenvalue of $\mathbb{T}_{0}$, meaning it has only one associated eigenvector. This implies that standard perturbation theory $\left(\omega^{(1)}=\mu \xi_{\mathbb{T}_{0}}^{L} \mathbb{T}^{(1)} \xi_{\mathbb{T}_{0}}^{R}\right.$, as is familiar from quantum mechanics) does not apply. Instead, we perturb the Jordan block,

$$
J=\left(\begin{array}{cc}
\omega_{0} & 1 \\
0 & \omega_{0}
\end{array}\right)=\left(p^{L} p^{R}\right)^{-1} p^{L} \mathbb{T}_{0} p^{R},
$$

where $p^{R}\left(p^{L}\right)$ is the right (left) generalized eigenvector block for $\omega_{0}$. Importantly, defective eigenvalues are particularly sensitive to perturbations (Kato 1995): if $\omega_{0}$ corresponds to an $n \times n$ Jordan block, then (in general) $\omega^{(1)} \sim \mathcal{O}\left(\mu^{1 / n}\right)$ (Moro \& Dopico 2002). Thus, the $\mathcal{O}(\mu)$ perturbation to $\mathbb{T}_{0}$ causes an $\mathcal{O}\left(\mu^{1 / 2}\right)$ perturbation to $\omega$.

We compute $\omega^{(1)}$ by calculating the eigenvalues of $\mathbb{T}_{0}+\mu \mathbb{T}^{(1)}$ in the generalized eigenvector basis (3). The result is particularly simple and useful:

$$
\omega=\omega_{0} \pm \mu^{1 / 2}\left[\left(\xi_{\mathcal{F}}^{L} \mathcal{T}_{F A}^{(1)} \xi_{\mathcal{A}}^{R}\right)\left(\xi_{\mathcal{A}}^{L} \mathcal{C} \xi_{\mathcal{F}}^{R}\right)\right]^{1 / 2}+\ldots
$$

which depends on the coupling terms $(\mathcal{C}$, coupling $\boldsymbol{a}$ to $\boldsymbol{f}$, and $\mathcal{T}_{F A}^{(1)}$, coupling $\boldsymbol{f}$ to $\boldsymbol{a}$ ) only through simple matrix multiplication. Noting that $\xi_{\mathcal{F}}^{L, R}, \xi_{\mathcal{A}}^{L, R}, \mathcal{C}$, and $\mathcal{T}_{F A}^{(1)}$ are, in general, complex, Equation (4) reveals why resonance instabilities are so virulent and so generic: the perturbation causes an instability $(\operatorname{Im}(\omega)>0)$ unless $\left(\xi_{\mathcal{F}}^{L} \mathcal{T}_{F A}^{(1)} \xi_{\mathcal{A}}^{R}\right)\left(\xi_{\mathcal{A}}^{L} C \xi_{\mathcal{F}}^{R}\right)$ is real and positive (or zero). Moreover, for $\mu \ll 1$, such modes grow more rapidly $\left(\operatorname{Im}(\omega) \sim \mathcal{O}\left(\mu^{1 / 2}\right)\right)$ than the usual perturbation theory expectation $(\operatorname{Im}(\omega) \sim \mathcal{O}(\mu))$.

At short wavelengths, the dust operator $\mathcal{A}$ itself becomes defective in $\omega_{0}$ (see Equation (10)), and we must generalize Equation (4) to three blocks:

$$
\mathbb{T}_{0}=\left(\begin{array}{ccc}
\mathcal{A}_{1} & \mathcal{C}_{12} & 0 \\
0 & \mathcal{A}_{2} & \mathcal{C}_{2 F} \\
0 & 0 & \mathcal{F}
\end{array}\right)
$$

where $\mathcal{A}_{1}, \mathcal{A}_{2}$, and $\mathcal{F}$ share an eigenvalue $\omega_{0}$. One obtains,

$$
\omega^{(1)}=s_{3} \mu^{1 / 3}\left[\left(\xi_{\mathcal{F}}^{L} \mathcal{T}_{F 1}^{(1)} \xi_{\mathcal{A}_{1}}^{R}\right)\left(\xi_{\mathcal{A}_{1}}^{L} \mathcal{C}_{12} \xi_{\mathcal{A}_{2}}^{R}\right)\left(\xi_{\mathcal{A}_{2}}^{L} \mathcal{C}_{2 F} \xi_{\mathcal{F}}^{R}\right)\right]^{1 / 3}+\ldots
$$

where $\mathcal{T}_{F 1}^{(1)}$ is lower left block of $\mathbb{T}^{(1)}$ and the values of $s_{3}=(1,-1 / 2 \pm i \sqrt{3} / 2)$ solve $s_{3}^{3}=1$. The perturbed system is always unstable for one $s_{3}$ unless Equation (6) is zero.

\section{Dust-Gas Systems}

We now specify $\mathcal{A}$ in Equation (1), modeling the grains as a pressureless fluid (Drew 1983; Jacquet et al. 2011), interacting with the gas fluid through a generic neutral drag force. The formalism is easily extended to incorporate more complex dust and drag physics (e.g., grain charge; Hopkins \& Squire 2018). We keep the fluid system (i.e., the $\mathcal{F}$ matrix) general at this stage, but assume it has density and velocity variables $\rho$ and $\boldsymbol{u}$ (in addition to other properties, e.g., magnetic field). We work in the frame where the fluid is stationary (which may have constant linear acceleration; Hopkins \& Squire 2017), with the grains streaming at velocity $\mathbf{w}_{s}=w_{s} \hat{\mathbf{w}}_{s}$.

On a homogenous background (with $\langle\cdot\rangle$ denoting a spatial average), the linearized and Fourier-decomposed continuum dust density, $\rho_{d}=\mu\langle\rho\rangle\left(1+\delta \rho_{d}\right)$, and velocity, $\boldsymbol{v}=\langle\boldsymbol{v}\rangle+\delta \boldsymbol{v}=\boldsymbol{w}_{s}+\delta \boldsymbol{v}$, 
satisfy

$$
\begin{gathered}
\left(-i \omega+i \mathbf{w}_{s} \cdot \boldsymbol{k}\right) \delta \rho_{d}+i \boldsymbol{k} \cdot \delta \boldsymbol{v}=0, \\
\left(-i \omega+i \mathbf{w}_{s} \cdot \boldsymbol{k}\right) \delta \boldsymbol{v}=-\delta \boldsymbol{F}_{\mathrm{drag}}\left(w_{s}, \boldsymbol{u}, \rho, \boldsymbol{v}\right) .
\end{gathered}
$$

Here $\delta \boldsymbol{F}_{\text {drag }}$ is the linearized drag acceleration, which we take as $\boldsymbol{F}_{\mathrm{drag}}=(\boldsymbol{v}-\boldsymbol{u}) / t_{s}$, where $t_{s}(\rho,|\boldsymbol{u}-\boldsymbol{v}|)$ is the "stopping time." We parameterize $t_{s}$ through $\delta t_{s} /\left\langle t_{s}\right\rangle=-\zeta_{s} \delta \rho /\langle\rho\rangle-$ $\zeta_{w} \hat{\mathbf{w}}_{s} \cdot(\delta \boldsymbol{v}-\delta \boldsymbol{u}) / w_{s}$, where $\left\langle t_{s}\right\rangle=t_{s}\left(\langle\rho\rangle, w_{s}\right)$. This form of the dust-fluid drag, determined by $\zeta_{s}$ and $\zeta_{w}$, encompasses many drag laws for uncharged grains in a polytropic fluid. For example, when the grain size $R_{d}$ is smaller than the gas meanfree path $\lambda_{\mathrm{mfp}}$ ("Epstein drag"; Epstein 1923),

$$
t_{s} \approx \frac{a_{\gamma}^{1 / 2} m_{d}}{\pi \rho c_{s} R_{d}^{2}}\left(1+a_{\gamma} \frac{|\boldsymbol{v}-\boldsymbol{u}|^{2}}{c_{s}^{2}}\right)^{-1 / 2}, \quad a_{\gamma} \equiv \frac{9 \pi \gamma}{128},
$$

which gives $\zeta_{s}^{\mathrm{Ep}}=\left(\gamma+1+2 \tilde{a}_{E}\right) /\left(2+2 \tilde{a}_{E}\right)$ and $\zeta_{w}^{\mathrm{Ep}}=\tilde{a}_{E} /$ $\left(1+\tilde{a}_{E}\right)$ (here, $\tilde{a}_{E} \equiv a_{\gamma}\left(w_{s} / c_{s}\right)^{2}, m_{d}$ is the mass of individual grains, and $\gamma$ is the fluid polytropic index). The coefficients $\zeta_{s}$ and $\zeta_{w}$ for other drag laws (e.g., Stokes or Coulomb drag; Draine \& Salpeter 1979) can be calculated in a similar manner (Hopkins \& Squire 2017). From momentum conservation, the drag on the fluid (contained in $\left.\mathbb{T}^{(1)}\right)$ is $+\left(\rho_{d} / \rho\right) \boldsymbol{F}_{\text {drag }}$.

With $\delta \boldsymbol{a}=\left(\delta \rho_{d}, \delta \boldsymbol{v}\right)$, Equations (7)-(8) give

$$
\mathcal{A}=\left(\begin{array}{cc}
\omega_{0} & \boldsymbol{k}^{T} \\
0 & \omega_{0} \mathbb{I}+\mathcal{D}_{\mathrm{drag}}
\end{array}\right), \quad \mathcal{C}=\left(\begin{array}{c}
0 \\
\mathcal{C}_{\boldsymbol{v}}
\end{array}\right),
$$

where $\omega_{0}=\boldsymbol{k} \cdot \mathbf{w}_{s}=k w_{s} \psi_{k w}$ is the resonant eigenmode (we define $\boldsymbol{k}=k \hat{\boldsymbol{k}}$ and $\psi_{k w} \equiv \hat{\boldsymbol{k}} \cdot \hat{\mathbf{w}}_{s}$ for convenience), $\mathcal{D}_{\text {drag }}=$ $-i\left(\mathbb{I}+\zeta_{w} \hat{\mathbf{w}}_{s} \hat{\mathbf{w}}_{s}^{T}\right) /\left\langle t_{s}\right\rangle$, and $\mathcal{C}_{\boldsymbol{v}}$ follows from the drag law (e.g., if $\left.\delta \boldsymbol{f}=(\delta \rho /\langle\rho\rangle, \delta \boldsymbol{u}), \mathcal{C}_{\boldsymbol{v}}=i\left(-\zeta_{s} \boldsymbol{w}_{s}, \mathbb{I}+\zeta_{w} \hat{\mathbf{w}}_{s} \hat{\mathbf{w}}_{s}^{T}\right) /\left\langle t_{s}\right\rangle\right)$. Evaluating Equation (4), we derive the RDI growth rate, which is valid when $\omega_{0}$ is also an eigenvalue of $\mathcal{F}$,

$$
\omega=\omega_{0} \pm i \mu^{1 / 2}\left[\left(\xi_{\mathcal{F}}^{L} \mathcal{T}_{\rho_{d}}^{(1)}\right)\left(\boldsymbol{k}^{T} \mathcal{D}_{\mathrm{drag}}^{-1} \mathcal{C}_{\boldsymbol{v}} \xi_{\mathcal{F}}^{R}\right)\right]^{1 / 2}+\ldots,
$$

where $\mathcal{T}_{\rho_{d}}^{(1)}=\mathcal{T}_{F A}^{(1)} \xi_{\mathcal{A}}^{R}=i \mathbf{w}_{s} /\left\langle t_{s}\right\rangle$ is the left column of $\mathcal{T}_{F A}^{(1)}$.

As $k$ increases, Equation (11) becomes invalid because $\mathcal{A}$ is nearly defective in $\omega_{0}$ when $\boldsymbol{k}^{T}$ dominates over $\mathcal{D}_{\text {drag. }}$. The theory is then modified to the triply defective case (6), which treats both $\mathcal{D}_{\text {drag }}$ and $\mu \mathbb{T}^{(1)}$ as perturbations. Using Equation (6) with $\mathcal{A}_{1}=\omega_{0}, \mathcal{A}_{2}=\omega_{0} \mathbb{I}, \mathcal{C}_{12}=\boldsymbol{k}^{T}, \xi_{\mathcal{A}_{2}}^{R}=\hat{\boldsymbol{k}}$, and $\mathcal{C}_{2 F}=\mathcal{C}_{\boldsymbol{v}}$, one obtains,

$$
\omega=\omega_{0}+s_{3} \mu^{1 / 3}\left[\left(\xi_{\mathcal{F}}^{L} \mathcal{T}_{\rho_{d}}^{(1)}\right)\left(\boldsymbol{k}^{T} \mathcal{C}_{\boldsymbol{v}} \xi_{\mathcal{F}}^{R}\right)\right]^{1 / 3}+\ldots
$$

for the "high- $k$ " RDI. From the characteristic polynomial of Equation (5), one finds that the transition between Equations (12) and (11) occurs when the two are approximately equal, at $\mu k \sim\left(\xi_{\mathcal{F}}^{L} \mathcal{T}_{\rho_{d}}^{(1)}\right)^{-1}\left(\hat{\boldsymbol{k}}^{T} \mathcal{C}_{\boldsymbol{v}} \xi_{\mathcal{F}}^{R}\right)^{2}\left(\hat{\boldsymbol{k}}^{T} \mathcal{D}_{\text {drag }}^{-1} \mathcal{C}_{\boldsymbol{v}} \xi_{\mathcal{F}}^{R}\right)^{-3}$.

Finally, we note that the result (11) is also not valid when $\omega_{0} \ll \mu w_{s}\left\langle t_{s}\right\rangle$ (i.e., when $\mu \mathbb{T}^{(1)}$ is larger than $\omega_{0}$ ), although instabilities generically persist in this regime (Hopkins \& Squire 2017). Inaccuracies can also arise near certain special points-e.g., when $\hat{\boldsymbol{k}}^{T} \mathcal{D}_{\text {drag }}^{-1} \mathcal{C}_{\boldsymbol{v}} \xi_{\mathcal{F}}^{R} \approx 0$-if the ordering used to derive Equations (11) and (12) becomes inaccurate. A small background dust pressure $\left\langle P_{d}\right\rangle$ causes the dust eigenmode to be weakly damped, $\operatorname{Im}\left(\omega_{0, \text { dust }}\right) \sim-\left\langle P_{d} / \rho_{d}\right\rangle k^{2}\left\langle t_{s}\right\rangle$, and our results are valid for $\omega^{(1)} \gg\left|\operatorname{Im}\left(\omega_{0, \text { dust }}\right)\right|$.

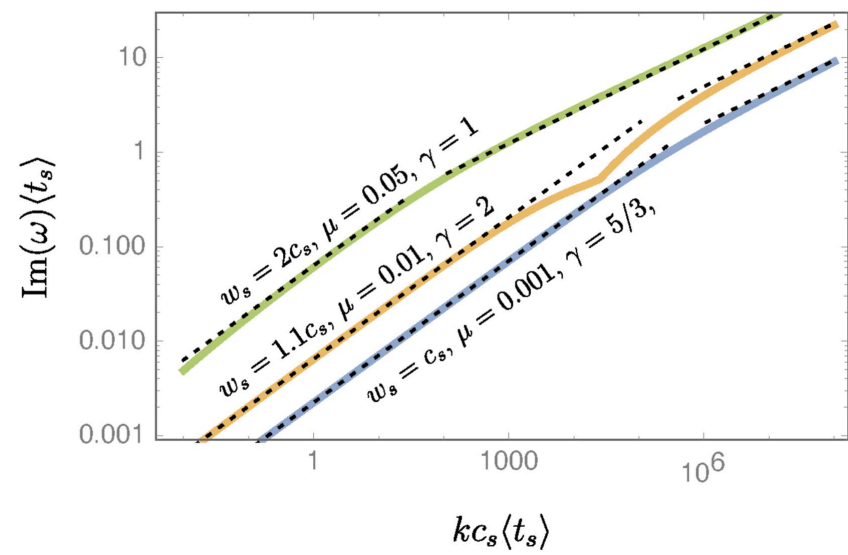

Figure 1. Acoustic RDI in hydrodynamics: resonant mode growth rate $\operatorname{Im}[\omega(k)]$, for several streaming velocities $w_{s} / c_{s}$ with $\psi_{k w}=\hat{\boldsymbol{k}} \cdot \hat{\boldsymbol{w}}_{s}=c_{s} / w_{s}$ (i.e., the resonant $\hat{\boldsymbol{k}}$ ). Thick colored lines show numerical solutions of the exact dispersion relation of the full coupled dust-gas system (Equations (7)-(8), coupled to the compressible Euler equations), and black dotted lines show analytic expressions (13)-(14). We use a variety of parameters as labeled, and Epstein drag (9) for $\zeta_{s}$ and $\zeta_{w}$. The dip around $k c_{s}\left\langle t_{s}\right\rangle \sim 10^{5}$ for $w_{s}=1.1 c_{s}$ occurs because the parameters $\left(\zeta_{s}=1.33, \zeta_{w}=0.35\right)$ lie near $\zeta_{s}=1+\zeta_{w}$ (see Equation (13) and Hopkins \& Squire 2017).

\section{Examples}

\subsection{Neutral Hydrodynamics}

We now consider the RDI in a variety of physical fluids (prescribing $\mathcal{F}$ ), starting with sound waves in compressible hydrodynamics. This amounts to setting $\mathcal{F}$ to describe a neutral compressible gas. This instability is explored in detail, including discussion of mode structure and astrophysical applications, in Hopkins \& Squire (2017). Noting the symmetry of the problem, we set $\hat{\mathbf{w}}_{s}=\hat{z}$ and consider 2D perturbations $\left(\boldsymbol{k}=k_{x} \hat{\boldsymbol{x}}+k_{z} \hat{z}\right)$. The linearized sound-wave eigenmodes for $(\delta \rho /\langle\rho\rangle$ and $\left.\delta u_{x}, \delta u_{z}\right)$ are $\xi_{\mathcal{F} \pm}^{R}=2^{-1 / 2}\left( \pm c_{s}^{-1}, k_{x} / k, k_{z} / k\right)^{T}$ and $\xi_{\mathcal{F}_{ \pm}}^{L}=$ $2^{-1 / 2}\left( \pm c_{s}, k_{x} / k, k_{z} / k\right)$, with eigenvalues $\omega_{ \pm}= \pm k c_{s}$. We see that for $w_{s}>c_{s}$ there is always a resonant mode-propagating in the direction $\psi_{k w}=k_{z} / k=c_{s} / w_{s}$-for which $\omega_{0}=\boldsymbol{k} \cdot \mathbf{w}_{s}=$ $k c_{s}$ for all $k$. The RDI growth rate thus increases indefinitely as $k \rightarrow \infty$ (neglecting viscosity, which damps the RDI once $k \gtrsim \lambda_{\mathrm{mfp}}^{-1}$ ).

Evaluating Equation (11), we obtain an approximate expression (to leading order in matrix perturbation theory) for this "acoustic RDI,"

$$
\omega \approx k c_{s}+s_{2} \mu^{1 / 2} k^{1 / 2}\left[\frac{c_{s}}{2\left\langle t_{s}\right\rangle}\left(1-\frac{\zeta_{s}}{1+\zeta_{w}}\right)\right]^{1 / 2},
$$

where $s_{2}= \pm(1+i) / \sqrt{2}$ solves $s_{2}^{2}=i$. For very highfrequency modes, Equation (12) gives

$$
\omega \approx k c_{s}+s_{3} \mu^{1 / 3} k^{1 / 3}\left[\frac{c_{s}}{2\left\langle t_{s}\right\rangle^{2}}\left(\zeta_{s}-1-\psi_{k w}^{2} \zeta_{w}\right)\right]^{1 / 3} .
$$

In Figure 1, we show several examples, comparing Equations (13)-(14) with direct numerical solutions of the exact linearized dust-gas dispersion relation for neutral, inviscid hydrodynamics and pressure-free grains coupled via Epstein drag. This confirms that the instabilities exist and shows that our analytic expressions are accurate where they apply. While the analytic Equations (13)-(14) are valid only at 
$k_{z} / k=c_{s} / w_{s}$, the system is also unstable at other mode angles and wavenumbers, albeit with lower growth rates $(\operatorname{Im}(\omega) \sim \mathcal{O}(\mu)$ when $\mu \ll 1$; Hopkins \& Squire 2017).

\subsection{Magnetohydrodynamics}

With more waves (Alfvén, slow, and fast modes), specifying $\mathcal{F}$ to describe MHD (including a magnetic field $\boldsymbol{B}$ in $\boldsymbol{f}$ ) allows for richer resonance phenomena. This structure, including the effects of grain charge (e.g., Lorentz forces on grains), is explored in detail in Hopkins \& Squire (2018), along with discussion of the diverse array of astrophysical environments where MHD RDIs could be important. As in hydrodynamics, MHD waves have constant phase velocities (for a given $\hat{\boldsymbol{k}}$ ), and the growth rate of the RDI increases indefinitely as $k \rightarrow \infty$. The resonant condition is $w_{s} \psi_{k w}=V_{\text {wave }}(\hat{\boldsymbol{k}})$ (where $V_{\text {wave }}$ is the wave phase velocity), and we take $\hat{\mathbf{w}}_{s}=\sin \theta_{w} \hat{\boldsymbol{x}}+\cos \theta_{w} \hat{z}$ with $\boldsymbol{B}=B_{0} \hat{z}$. Remarkably, because the slow mode phase velocity approaches zero as $\hat{\boldsymbol{k}} \cdot \boldsymbol{B} \rightarrow 0$, an instability occurswith $\operatorname{Im}(\omega) \rightarrow \infty$ as $k \rightarrow \infty$-for any $w_{s}$, so long as $\theta_{w} \neq 0$.

Evaluating (11), we find that Alfvén waves do not cause a mid- $k$ RDI (the product in square brackets is zero for neutral grains), while resonance with slow or fast waves triggers the "magnetosonic RDI,"

$$
\begin{aligned}
\omega_{ \pm} \approx & k v_{ \pm} c_{s}+s_{2}\left(\frac{k \mu c_{s}}{\left\langle t_{s}\right\rangle}\right)^{1 / 2} \\
& \times\left[\frac{\tilde{\zeta}_{w}-\zeta_{s}}{\tilde{\zeta}_{w}}\left(\frac{v_{\mp}^{2}}{\psi_{k w}} \cos \theta_{w}-\frac{k_{z}}{k}\right) \Theta_{ \pm}\right]^{1 / 2} .
\end{aligned}
$$

Here $v_{+}=v_{F} / c_{s}$ and $v_{-}=v_{S} / c_{s}$ are the normalized fast and slow phase velocities, $\tilde{\zeta}_{w}=1+\zeta_{w}$, and

$$
\Theta_{ \pm} \equiv \frac{k}{k_{z}} \frac{v_{ \pm}^{3}\left(1-v_{\mp}^{2}\right)}{\left(1-v_{\mp}^{2}\right)^{2}+v_{ \pm}^{2}\left(1-2 v_{\mp}^{2}\right)+\frac{k_{\perp}^{2} v_{A}^{2}}{k^{2} c_{s}^{2}}+\frac{k^{2}}{k_{z}^{2}} v_{\mp}^{4} v_{ \pm}^{2}},
$$

with $v_{A} \equiv B_{0} / \sqrt{4 \pi\langle\rho\rangle}$. The high- $k$ form (Equation (12)) of the magnetosonic RDI is similar, but we omit it here (the Alfvén wave is also destabilized at high $k$; see Hopkins \& Squire 2018). In Figure 2, we compare these analytic results to numerical solutions of the full tenth-order grain-fluid dispersion relation, for a variety of magnetosonic resonances at different angles.

\subsection{Stratified Fluid}

Our final example is a stratified adiabatic fluid, within the Boussinesq approximation. This instability, particularly its application to planetesimal formation in disks, is treated in detail in Squire \& Hopkins (2017). With background gas stratification $\nabla \ln \left(p_{0} \rho_{0}^{-5 / 3}\right)=-(5 / 3) L_{\rho}^{-1} \hat{z}$ and gravitational force $\boldsymbol{g}=g \hat{z}=$ $\tilde{g} \hat{z}+\mathcal{O}(\mu)$ (where $\left.\tilde{g} \equiv \rho_{0}^{-1} d p_{0} / d z\right)$, the linearized fluid equations for perturbations $\delta \boldsymbol{u}, \delta \bar{\rho}=\delta \rho /\langle\rho\rangle$, and $\delta \bar{T}=\delta T /\langle T\rangle$ (temperature) are (Goldreich \& Schubert 1967; Balbus 1995),

$$
\begin{aligned}
& \partial_{t} \delta \bar{\rho}+L_{\rho}^{-1} \delta u_{z}=0, \quad \delta \bar{\rho}+\delta \bar{T}=0, \\
& \partial_{t} \delta \boldsymbol{u}=-\langle\rho\rangle^{-1} \nabla \delta p+\tilde{g} \delta \bar{\rho} \hat{z}, \quad \nabla \cdot \delta \boldsymbol{u}=0,
\end{aligned}
$$

where $\delta p$ enforces $\nabla \cdot \delta \boldsymbol{u}=0$. The system supports oscillations at $\omega_{0}= \pm\left(k_{\perp} / k\right) N_{\mathrm{BV}}$, where $N_{\mathrm{BV}}=\sqrt{\tilde{g} / L_{\rho}}$ is the BruntVäisälä frequency and $k_{\perp}^{2}=k_{x}^{2}+k_{y}^{2}$. We set $\hat{\mathbf{w}}_{s}=\sin \theta_{w} \hat{\boldsymbol{x}}+$



Figure 2. Magnetosonic RDI in MHD: blue (orange) lines show numerically calculated RDI growth rates for grains resonant with the fast (slow) mode with streaming angle $\theta_{w}=45^{\circ}$ and $\mu=0.01 \quad\left(\mu=0.05\right.$ for the $w_{s}=10 c_{s}$ fast wave). Dotted lines show the analytic predictions (15) (Equation (12) at high $k$ ). In each case, we calculate $\zeta_{s}$ and $\zeta_{w}$ assuming Epstein drag (9) with $\gamma=5$ / 3. The resonant mode directions, $\hat{\boldsymbol{k}}=(\cos \phi \sin \theta, \sin \phi \sin \theta, \cos \theta)$, are $\theta=70^{\circ}, \phi=49^{\circ} .6$ (slow wave, $w_{s}=0.1 c_{s}$ ) $; \theta=40^{\circ}, \phi=108^{\circ} .4$ (slow wave, $\left.w_{s}=1.5 c_{s}\right) ; \theta=20^{\circ}, \phi=63^{\circ} .5$ (fast wave, $w_{s}=1.5 c_{s}$ ); $\theta=30^{\circ}, \phi=57^{\circ} .6$ (fast wave, $w_{s}=10 c_{s}$ ). The low-k discrepancy of the fast-wave prediction at $w_{s}=10 c_{s}$ is due to an additional instability.

$\cos \theta_{w} \hat{z}$, and resonance occurs when $k_{\text {res }} w_{s} \psi_{k w}=\left(k_{\perp} / k\right) N_{\mathrm{BV}}$. There is now only one $k_{\text {res }}$ (for $w_{s}$ and $\hat{\boldsymbol{k}}$ given), because $V_{\text {wave }} \propto N_{\mathrm{BV}} / k$. We assume Epstein drag (9), which-using $\delta \rho+\delta T=0$ and $w_{s} \ll c_{s}$-implies $\delta t_{s} /\left\langle t_{s}\right\rangle \approx-\delta \bar{\rho} / 2$.

Inserting Equation (17) and $k=k_{\text {res }}$ into Equation (11), we obtain the "Brunt-Väisälä RDI,"

$$
\omega \approx \frac{k_{\perp}}{k} N_{\mathrm{BV}}+i \mu^{1 / 2} N_{\mathrm{BV}}\left[\frac{w_{s}}{4 \tilde{g}\left\langle t_{s}\right\rangle}\left(\cos \theta_{w}-\frac{k_{z}}{k} \psi_{k w}\right)\right]^{1 / 2}
$$

(the high- $k$ scaling (12) is never physically applicable). Evidently, the RDI is unstable, around $k_{\text {res }}=k_{\perp} N / \mathbf{w}_{s} \cdot \boldsymbol{k}$, unless grains stream exactly against gravity $\left(\theta_{w}=\pi\right.$ if $\left.L_{\rho}>0\right)$. In Figure 3 , we compare numerical solutions with Equation (18) for gravitationally settling grains $\left(\boldsymbol{w}_{s}=\boldsymbol{g}\left\langle t_{s}\right\rangle\right)$, showing the agreement at $k=k_{\text {res }}$. A compressible treatment reveals minor corrections to Equation (18) from corrections to the Boussinesq approximation; ${ }^{3}$ see Squire \& Hopkins (2017).

Discussion-We have shown that dust grains streaming (with velocity $\mathbf{w}_{s}$ ) through a fluid are usually unstable. Specifically, an RDI occurs whenever the dust streaming frequency $\boldsymbol{k} \cdot \mathbf{w}_{s}$ matches the frequency of a fluid wave $\omega_{0}(\boldsymbol{k})$, except for pathological forms of the dust-to-fluid coupling (see Equations (11)-(12)). All RDIs generically cause grains to clump spatially as they grow and will also seed turbulence if sufficiently strong. This could have potentially important consequences for a wide variety of astrophysical regions and processes, including planetesimal formation, cool-star winds, AGN torii and winds, starburst regions, HII regions, supernova ejecta, and the circumgalactic medium. Extended discussion of these implications follows in Hopkins \& Squire $(2017,2018)$ and Squire \& Hopkins (2017).

Rather than exploring any one family of RDIs in detail, our purpose here has been to demonstrate the existence of RDIs

\footnotetext{
3 The local treatment also requires $\mu^{1 / 2} \gg\left(k L_{\rho}\right)^{-1}$, so that the effect of grains is larger than corrections to the gas modes.
} 


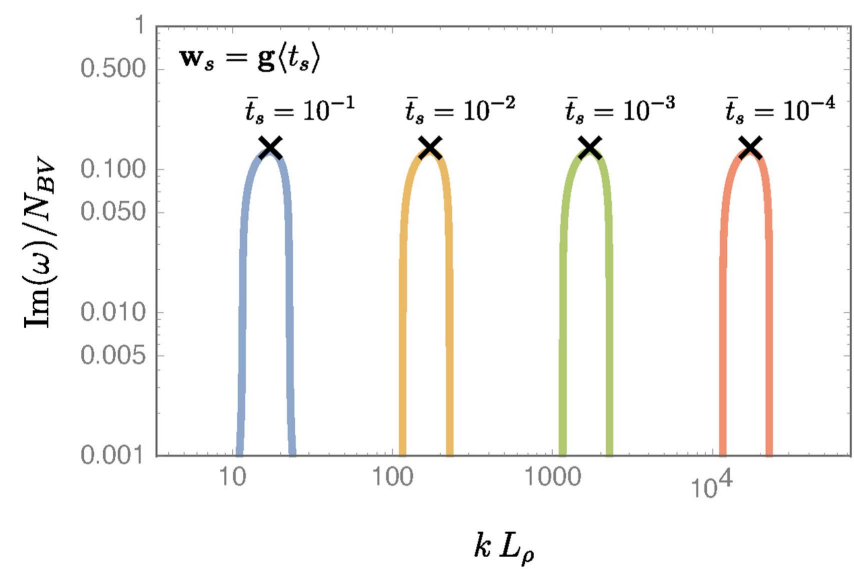

Figure 3. Brunt-Väisälä RDI in a stratified fluid: solid lines show numerically calculated growth rates for differently sized grains, specified by the normalized stopping time $\bar{t}_{s} \equiv\left\langle t_{s}\right\rangle N_{\mathrm{BV}}$. We set $\boldsymbol{w}_{s}$ to the "natural" settling of grains due to gravity, $\boldsymbol{w}_{s}=\boldsymbol{g}\left\langle t_{s}\right\rangle\left(\theta_{w}=0\right)$, assume Epstein drag (9) with $\mu=0.1$, and set $k_{z}=k / 2, k_{\perp}=\sqrt{3 / 4} k$. The black crosses show the RDI (18) at resonance, $k=k_{\text {res }}$. Smaller grains excite smaller-scale oscillations because they settle more slowly $\left(V_{\text {wave }} \propto N_{\mathrm{BV}} / k \propto w_{s}\right)$, but $\operatorname{Im}(\omega)$ is independent of $\left\langle t_{s}\right\rangle$ when $w_{s} \propto\left\langle t_{s}\right\rangle$. Because grains move through the atmosphere over a timescale $t_{\text {settle }} \sim L_{\rho} / w_{s}$, the RDI grows sufficiently fast to clump grains (as observed in Lambrechts et al. 2016) if $\operatorname{Im}(\omega) / N_{\mathrm{BV}} \gtrsim \bar{t}_{s}=\left\langle t_{s}\right\rangle N_{\mathrm{BV}}$.

and provide an algorithm for identifying the different variants. However, for the sake of illustration, we have provided several examples of the RDI in different fluid systems. In hydrodynamics and MHD, the constant phase velocity of linear waves $\left(\omega_{0} \propto k\right)$ implies that RDI growth rates increase indefinitely as $k \rightarrow \infty$, in the absence of viscosity or resistivity. In MHD, slow waves are destabilized for any $w_{s}=\left|\mathbf{w}_{s}\right|$ (Figure 2); in hydrodynamics, sound waves are destabilized whenever $w_{s}>c_{s}$ (Figure 1). Our final example-a stratified fluid-illustrates the RDI with Brunt-Väisälä oscillations and shows that small grains settling through a stratified atmosphere are unstable. Extensions to other systems (e.g., other fluids or charged grains) are straightforward, given the simplicity of the perturbed eigenvalues (4). For example, as shown in Squire \& Hopkins (2017), the maximum growth rate of the well-known disk "streaming instability" (Youdin \& Goodman 2005) at $\mu<1$ can be calculated as the "epicyclic RDI" using Equation (11).

Let us finish by reiterating the algorithm presented here for finding drag-induced instabilities in dust-laden fluids: match $\boldsymbol{k} \cdot \mathbf{w}_{s}$ to an oscillation mode (wave) of the fluid; Equation (11) or (12) then says that the system is most likely unstable, and gives the growth rate of the resonant drag modes.

We thank A. R. Bell, J. W. Burby, E. Quataert, and E. S. Phinney for enlightening discussions. Support for J.S. \& P.F.H. was provided by an Alfred P. Sloan Research Fellowship, NASA ATP grant NNX14AH35G, and NSF Collaborative Research grant \#1411920 and CAREER grant \#1455342. J.S. was funded in part by the Gordon and Betty Moore Foundation through grant GBMF5076 to Lars Bildsten, Eliot Quataert, and E. Sterl Phinney.

\section{ORCID iDs}

J. Squire (i) https://orcid.org/0000-0001-8479-962X

P. F. Hopkins (1) https://orcid.org/0000-0003-3729-1684

\section{References}

Armitage, P. J. 2011, ARA\&A, 49, 195

Balbus, S. A. 1995, ApJ, 453, 380

Chiang, E., \& Youdin, A. N. 2010, AREPS, 38, 493

Childress, W. S., \& Spiegel, E. A. 1975, SIAMR, 17, 136

Deguchi, S. 1997, in IAU Symp. 180, Planetary Nebulae, ed. H. J. Habing \& H. J. G. L. M. Lamers (Dordrecht: Kluwer), 151

Dobson, I., Zhang, J., Greene, S., Engdahl, H., \& Sauer, P. W. 2001, ITCS, 48,340

Dominik, C., Gail, H.-P., \& Sedlmayr, E. 1989, A\&A, 223, 227

Draine, B. T. 2004, in The Cold Universe, ed. A. W. Blain et al. (Dordrecht: Springer), 213

Draine, B. T., \& Salpeter, E. E. 1979, ApJ, 231, 77

Drew, D. A. 1983, AnRFM, 15, 261

Epstein, P. S. 1923, PhRv, 22, 1

Ferrière, K. M. 2001, RvMP, 73, 1031

Franco, J., Ferrini, F., Barsella, B., \& Ferrara, A. 1991, ApJ, 366, 443

Gilman, R. C. 1972, ApJ, 178, 423

Goldreich, P., \& Schubert, G. 1967, ApJ, 150, 571

Goldreich, P., \& Ward, W. R. 1973, ApJ, 183, 1051

Goodman, J., \& Pindor, B. 2000, Icar, 148, 537

Hopkins, P. F., Hayward, C. C., Narayanan, D., \& Hernquist, L. 2012, MNRAS, 420, 320

Hopkins, P. F., \& Squire, J. 2017, arXiv:1707.02997

Hopkins, P. F., \& Squire, J. 2018, arXiv:1801.10166

Jacquet, E., Balbus, S., \& Latter, H. 2011, MNRAS, 415, 3591

Johansen, A., Oishi, J. S., Mac Low, M.-M., et al. 2007, Natur, 448, 1022

Kato, T. 1995, Perturbation theory for linear operators, Grundlehren der Mathematischen Wissenschaften (Berlin: Springer)

Käufl, H. U., \& Siebenmorgen, R. 1996, The Role of Dust in the Formation of Stars (Berlin: Springer)

Kennel, C. F., \& Wong, H. V. 1967, JPIPh, 1, 75

Kirillov, O. 2013, Nonconservative Stability Problems of Modern Physics, De Gruyter Studies in Mathematical Physics (Berlin: De Gruyter)

Krein, M. 1950, Dok. Akad. Nauk SSSR, 73, 445

Krolik, J. H., \& Begelman, M. C. 1988, ApJ, 329, 702

Krüger, H., Strub, P., Grün, E., \& Sterken, V. J. 2015, ApJ, 812, 139

Lambrechts, M., Johansen, A., Capelo, H. L., Blum, J., \& Bodenschatz, E. 2016, A\&A, 591, A133

Mastrodemos, N., Morris, M., \& Castor, J. 1996, ApJ, 468, 851

Moro, J., \& Dopico, F. M. 2002, Applied Mathematics and Scientific Computing (New York: Springer)

Morris, M. 1993, in European Southern Observatory Conf. and Workshop Proc. 46, Mass Loss on the AGB and Beyond, ed. H. E. Schwarz (Garching: ESO), 60

Murray, N., Quataert, E., \& Thompson, T. A. 2005, ApJ, 618, 569

Nakagawa, Y., Sekiya, M., \& Hayashi, C. 1986, Icar, 67, 375

Netzer, N., \& Elitzur, M. 1993, ApJ, 410, 701

Norris, B. R. M., Tuthill, P. G., Ireland, M. J., et al. 2012, Natur, 484, 220

Pumir, A., \& Wilkinson, M. 2016, ARCMP, 7, 141

Rao, N., Shukla, P., \& Yu, M. 1990, P\&SS, 38, 543

Saffman, P. G. 1962, JFM, 13, 120

Shukla, P. K. 2001, PhPl, 8, 1791

Squire, J., \& Hopkins, P. F. 2017, arXiv:1711.03975

Sundaresan, S. 2003, AnRFM, 35, 63

Thompson, T. A., Quataert, E., \& Murray, N. 2005, ApJ, 630, 167

Tytarenko, P. V., Williams, R. J. R., \& Falle, S. A. E. G. 2002, MNRAS, 337, 117

Verscharen, D., Bourouaine, S., \& Chandran, B. D. G. 2013, ApJ, 773, 163

Weingartner, J. C., \& Draine, B. T. 2001, ApJS, 134, 263

Youdin, A. N., \& Goodman, J. 2005, ApJ, 620, 459 